\title{
The Metallicity Evolution of Galaxies through the Cosmic Epochs
}

\author{
R. Maiolino ${ }^{1}$ \\ ${ }^{1}$ INAF - Osservatorio Astronomico di Roma, \\ via di Frascati 33, Monte Porzio Catone (RM), 00040, Italy \\ email: maiolino@oa-roma.inaf.it
}

\begin{abstract}
We have found that local galaxies follow a very tight relation between gas metallicity, stellar mass and SFR, suggesting that their evolution is characterized by a long standing equilibrium between gas inflows, outflows and star formation. Surprisingly, even distant galaxies, out to $\mathrm{z}<2.5$, follow the same relation, suggesting that the same dominant mechanism of galaxy evolution is in place at any epoch, out to $\mathrm{z}<2.5$. However, by using deep near-IR spectroscopy (probing optical nebular lines at high-z), we find that galaxies at $\mathrm{z}>3$ deviate from such fundamental relation, by being significantly more metal poor. Spatially resolved metallicity maps of $\mathrm{z}>3$ disk galaxies reveal that they are characterized by central regions with low metallicity associated with the peak of star formation, indicating that the latter is due to massive inflow of pristine gas that both boosts star formation and dilutes the gas metallicity. Overall these results suggest that the metallicity evolution of galaxies at $z>3$ is due to an excess of gas inflow at such early epochs, as expected by some recent models. Finally, we investigate the metallicity of merging systems, both locally and at high-z. By exploiting recent Herschel data, we have found that in these systems the dust content directly measured through the FIR-submm data is much higher than inferred from the metallicity measured through the optical nebular lines. The latter result suggests that, in these heavily obscured systems, optical observations only probe the outer, less enriched regions and are not representative of the bulk of the metal content.
\end{abstract}

Keywords. galaxies: abundances, galaxies: active, galaxies: evolution, ISM: dust

The metal content of galaxies depends on their star formation history, as well as on the effects of pristine gas inflows and metal enriched outflows. These are all phenomena regulating the evolution of galaxies. As a consequence, tracing the metallicity of galaxies through the cosmic epochs is a fundamental tool to investigate the mechanisms driving galaxy evolution. In the local universe galaxies follow a well defined relationship between metallicity (both in the gas phase and in stars) and stellar mass, with more massive galaxies being more metal rich. Such a mass-metallicity relation is regarded as a reference that any model of galaxy formation has to reproduce. However, more recently we have found that the gas metallicity in local galaxies is not only related to the stellar mass, but also to the star formation rate (SFR). More specifically, at a given stellar mass the gas metallicity decreases with increasing SFR. This anticorrelation can be interpreted in terms of pristine gas inflows that both increase the SFR (as a consequence of the Schmidt-Kennicutt relation) and dilute the gas metallicity. More generally, gas metallicity, stellar mass and SFR, describe a tight three-dimensional relation (Fig. 1) with a very small dispersion of 0.05 dex in metallicity (Mannucci et al. 2010). This tight relationship suggests that the evolution of galaxies is regulated by a long standing equilibrium between star formation, gas inflows and outflows (e.g. Bouchè et al. 2010, Finaltor \& Davè 2008).

Within this context, a very interesting result is that also high-z galaxies, out to $\mathrm{z}<2.5$, appear to follow the same fundamental relation between metallicity, mass and SFR 

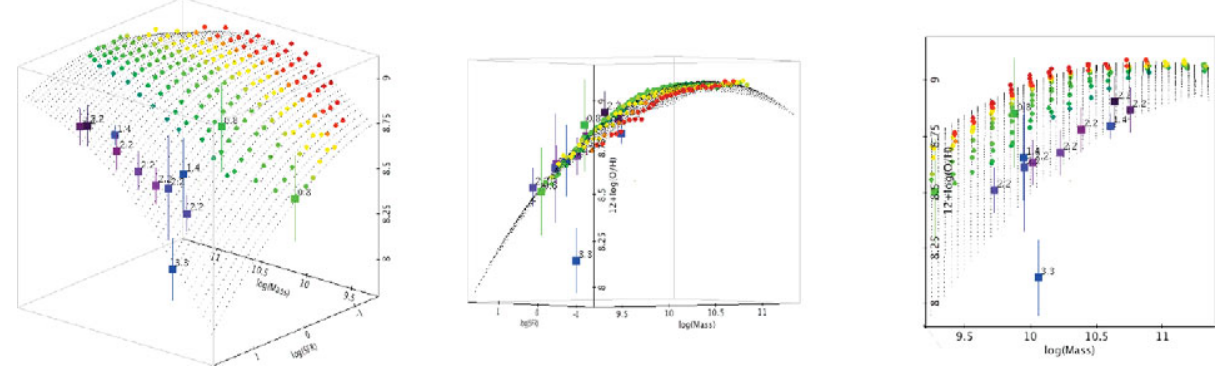

Figure 1. Different projections of the fundamental metallicity relation between gas metallicity, stellar mass and star formation rate for local galaxies. The small points, with different colors, indicate different star formation rates. Squares with error bars indicate the average location of distant star forming galaxies at different redshifts (the latter indicated by the number associated with each point). From Mannucci et al. (2010).

(Fig. 1). This result suggests that the same dominant mechanism of galaxy formation is at work through all cosmic epochs, within the redshift range $0<\mathrm{z}<2.5$. However, we do find a significant offset in metallicity for galaxies at $\mathrm{z} \sim 3.5$ (being more metal poor by 0.6 dex than lower redshift galaxies with the same mass and SFR), as inferred from the measurements of the nebular emission lines in about 40 galaxies from our AMAZE and LSD surveys performed with SINFONI at ESO (Maiolino et al. 2008, Mannucci et al. 2009, Maiolino et al. 2010). These results suggest that the real epoch of evolution of galaxy, in terms of change in galaxy formation mechanism, occurs only at $\mathrm{z}>3$.

Such "evolution" at $z>3$ could be due to an increased role of galaxy merging, which may gravitationally destabilize metal poor gas in the galaxy outskirts and drive it towards the central regions, where it participates to star formation and dilutes the pre-existing gas. The integral field spectroscopic information obtained with our SINFONI data at $\mathrm{Z} \sim 3.5$ allows us to investigate the dynamics of these galaxies and, therefore, test the merging scenario. We find that about one third of the galaxies in our sample (especially the massive ones) show regular rotation patterns, without any indication of merging (Gnerucci et al. 2011). This finding indicates that at $\mathrm{z} \sim 3.5$ massive rotating galaxy disks are already in place, although highly turbulent (with a velocity dispersion even higher than the rotational velocity). About one third of the galaxies does show irregular kinematics, suggestive of recent or ongoing merging, while for one third of the galaxies we cannot infer unambiguous information (due either to lack of spatial resolution or low signal-to-noise ratio). In terms of metallicity, we obtain that, at $\mathrm{z} \sim 3.5$, there is no difference between merging systems and rotating disks: both classes of objects deviate by similar amounts from the fundamental relation between metallicity, mass and SFR characterizing lower redshift galaxies (Troncoso et al., in prep.). This result indicates that an increased role of galaxy merging cannot be the only effect responsible for the metallicity evolution observed at $\mathrm{z}>3$.

Independent information on the phenomena occurring at $\mathrm{z}>3$ comes from the analysis of metallicity gradients. Indeed, our integral field spectra allow us to spatially resolve the nebular lines ratios, and therefore map the metallicity in a few galaxies. In particular, metallicity gradients have been resolved in a few massive disk galaxies at $\mathrm{z}>3.5$ characterized by regular rotation (Fig. 2, Cresci et al. 2010). We find that these galaxies, in contrast with the bulk of local galaxies, are characterized by minimum of the gas metallicity located close in the central regions and corresponding to the peak of star formation activity. We interpret this result as evidence for cold flows of pristine gas that both boost star formation and locally dilute the gas metallicity. A similar investigation on other 
galaxies in the AMAZE and LSD samples reveals that this phenomenon is common to most of the galaxies at $\mathrm{z} \sim 3.5$ (Troncoso et al., in prep.). As a consequence, we identify the main origin of the chemical evolution observed at $z>3$ in an increased rate of cold flows of pristine gas at such early epochs, as expected by some recent theoretical models (e.g. Dekel et al. 2009).
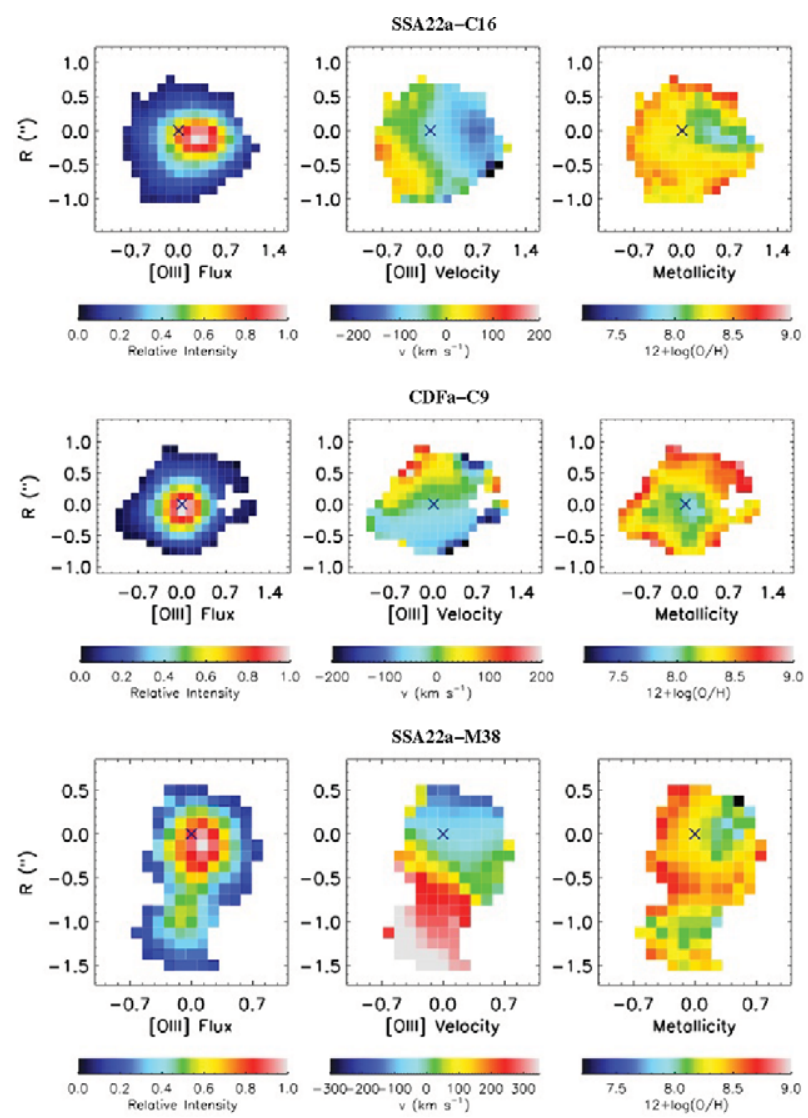

Figure 2. Surface brightness of the [OIII] $\lambda 5007$ line, velocity map, and gas phase metallicity, for three rotating disk galaxies at $\mathrm{z} \sim 3.5$ (Cresci et al. (2010)). Low metallicity regions are associated with the peaks of star formation. The crosses mark the position of the continuum peak.

Finally, we specifically focus on the case of dusty merging systems, both in the local universe and at high redshift. It has been shown that local Luminous and Ultraluminous Infrared Galaxies (LIRGs and ULIRGs), generally hosted in interacting and merging systems, are characterized by a metallicity, as inferred from the optical nebular lines, much lower than disk galaxies with the same mass (Rupke et al. 2008), even lower than expected by the fundamental relation between metallicity, mass and SFR discussed above. The same strong metal deficiency has been observed in distant sub-millimeter galaxies (SMGs, Santini et al. 2010), which are also hosted in merging systems. This phenomenon has been interpreted as a consequence of inflows of metal poor gas towards the central regions due to gravitational torques and tidal effects during galaxy interactions. However, recent Herschel data have revealed a different picture. More specifically, with the aid of Herschel photometric data we could measure the dust content of high-z SMGs and in local ULIRGs (Santini et al. 2010). Since more than half of metals in the ISM are locked 
into dust grains one would expect that the dust content mirrors the gas metallicity, i.e. the dust content of ULIRGs and SMGs should be low. On the contrary, we find that, for a given stellar mass, the dust mass of both local ULIRGs and high-z SMGs is much higher than in normal disk galaxies (Fig. 3). More in detail, for those ULIRGs and SMGs having CO observations, we derive that the dust-to-gas ratio is much higher than expected from the gas metallicity (Santini et al. 2010). The simpler explanation for this paradoxical result is that in these very dusty, obscured systems the optical nebular lines used to measure the metallicity only probe the outer, less enriched, regions of the galaxy, not being representative of the bulk of the metallicity in the central regions. As a consequence, much care should be adopted when inferring the metallicity of such heavily obscured merging systems by exploiting optical diagnostics, both in local systems and at high-z; the global content of metals given by the dust mass inferred from FIR-submm data (in particular from Herschel and, soon, from ALMA), is probably a much safer and more accurate tool.

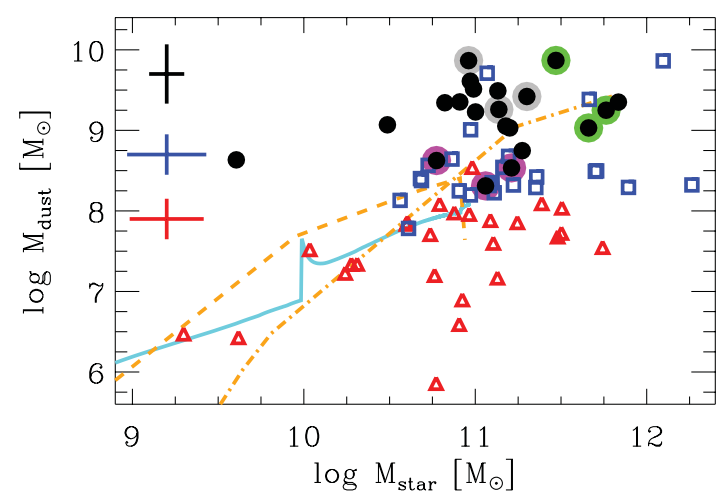

Figure 3. Dust mass versus stellar mass for local ULIRGs (blue squares) and $\mathrm{z} \sim 2$ SMGs (black circles), compared with local spiral galaxies (red triangles). From Santini et al. (2010)

These results have been obtained thanks to the efforts of a wide collaboration, involving the AMAZE and LSD teams, as well as the Herschel-PEP consortium.

\section{References}

Bouchè, N., et al. 2010, ApJ, 718, 1001

Cresci, G., et al. 2010, Nature, 467, 811

Dekel, A., et al. 2009, Nature, 457, 451

Finlator, K., \& Davè 2008, MNRAS, 385, 2181

Gnerucci, A., et al. 2010, A\&A, in press (arXiv:1007.4180)

Maiolino, R., et al. 2008, A\&A, 30, 490

Maiolino, R., et al. 2010, The Messenger, 142, 36

Mannucci, F., Cresci, G., Maiolino, R., Marconi, A., \& Gnerucci, A. MNRAS, 408, 2115

Mannucci, F., et al. 2009 MNRAS, 398, 1915

Rupke, D. S. N., et al. 2008, ApJ, 674, 172

Santini, P., et al. 2010, AछA, 518, L154 